\title{
Load Matrix Optimization in Mobile Cellular Networks
}

\author{
Jihun Jung ${ }^{1}$, Mohammad Abaii ${ }^{2}$, Rahim Tafazolli ${ }^{3}$ \\ Center for Communication System Research (CCSR) \\ University of Surrey \\ Guildford, Surrey, GU2 7XH, United Kingdom \\ joe.jihun.jung@googlemail.com ${ }^{1}$ \\ $\left\{\right.$ m.abaii ${ }^{2}$, r.tafazolli $\left.{ }^{3}\right\} @$ surrey.ac.uk
}

\begin{abstract}
Future mobile networks providing multitude of services with large range of $\mathrm{QoS}$ requirements has made the importance of Radio Resource Management (RRM) ever more significant. Traditional scheduling schemes have approached resource allocation mainly from a cell point of view to large extent ignoring effects of multi-cell architecture. Recently, Load Matrix (LM) concept has been proposed which facilitates joint management of interference within and between cells for allocation of radio resources. In this paper, we assess the Load Matrix scheduling performance when combined with traditional schedulers. In addition, a new LM algorithm for efficient RRM, called Global Proportional Fair (GPF) is proposed. The results show that GPF algorithm with an appropriate window size outperforms traditional scheduling schemes in terms of both throughput and fairness. It combines the throughput advantage of Max C/I and the fairness advantage of Proportional Fair scheduler.
\end{abstract}

Keywords-radio resource ranagement; power management; load matrix; interferece management; HSUPA

\section{INTRODUCTION}

With growing demand for wireless communications, advanced mobile cellular systems have evolved in many countries including in Europe, America, Korea, Japan and China. The maximization of revenue per bandwidth is one key factor for emerging systems given the limitation of radio resources available. On the other hand, customers demand more bandwidth and higher data transmission rates in order to support multimedia contents and real-time services.

In order to satisfy this demand, efficient resource allocation and scheduling must be adopted to maintain the reasonable QoS and to increase the spectrum utilization. Wireless channel condition is affected by many factors such as fading, shadowing and interference which degrade the quality of the signal and cause delay. These factors will eventually affect the total capacity of the system. In addition, two forms of interference i.e. inter-cell interference that occurs between cells and intra-cell interference caused by own users can be distinguished.

In uplink, resource allocation schemes can be divided in two categories namely distributed and centralised resource allocation. One of the main objectives of the distributed allocation is to reduce the complexity to the RNC (Radio Network Controller) by calculating the resource allocation factors of every base station (BS) with the fact that it does not know the channel conditions of adjacent cells. In the centralised allocation, the network controller is responsible for allocating the resources in every cell. The main drawback of the distributed allocation scheme in UMTS (Universal Mobile Terrestrial System) for instance, is that the interference caused by other cells can severely degrade the signal strength due to lack of information about adjacent cells.

Scheduling plays a very important role in acquisition of higher capacity through rate assignment and power allocation. There are a number of schedulers studied and deployed such as Round Robin, Max C/I [1], Proportional Fair [2][3] and Score-Based [4] as well as opportunistic algorithms.

In uplink, users have their own transmitter with power restrictions which requires complex parallel power scheduling to each user at all times. Meanwhile, BS needs to consider cell load contributed by its own users as well as other cells. If users transmit without appropriate power allocation, it will affect others as a form of intercell or intracell interference and increase the load (Noise Rise) to neighbouring cells degrading spectral efficiency. Therefore, proper interference management is vital in the overall resource utilization in the network.

A different approach towards scheduling, addressing interference problem is Load Matrix. It is also a priority-based approach which deals with the user priority and its contribution to RoT (Rise over Thermal noise) at every BS [5]. This paper introduces a new optimized algorithm for the priority function in Load Matrix that can be used to achieve efficient control of interference. In order to attain the optimized priority function in Load Matrix, a novel algorithm called the Global Proportional Fair (GPF) is proposed. The discussion provided in this paper will also include the performance benefit compared to the traditional algorithms. HSUPA (High Speed Uplink Packet Access) system [6] has been used as the simulation environment to evaluate the performance.

The provision of wireless resource management, its main problems and objectives are discussed in section II. Section III presents traditional scheduling algorithms with and without Load Matrix enhancement, as well as the GPF proposed as an optimized LM algorithm. Simulation results are presented and the achievements are discussed in section IV. Finally, section $\mathrm{V}$ concludes the paper. 


\section{RESOURCE AlLOCATION IN UPLINK}

Assessing the quality of resource allocation, one can distinguish two types of merits. One is overall throughput i.e. summation of cell capacity achieved in the system. The other is fairness amongst users in terms of offered transmission opportunities, where the location of user is likely to be distributed in a uniform manner over the whole network and the network controller needs to maintain the service level agreement. The trade-off between throughput and fairness is very important in a scheduling algorithm.

One of the main objectives in resource allocation and scheduling is to comprise available resources and constraint elements in an efficient way. It is shown in [5] that finding optimum scheduling in a wireless network environment is a NP-hard problem. Therefore iterative approaches [7][8] are often considered to obtain the best performance that can be achieved.

The main challenge in uplink scheduling comes from intercell interference and how to deal with it. Fig. 1 illustrates RoT fluctuation in a cell due to intercell interference using a traditional scheduler.

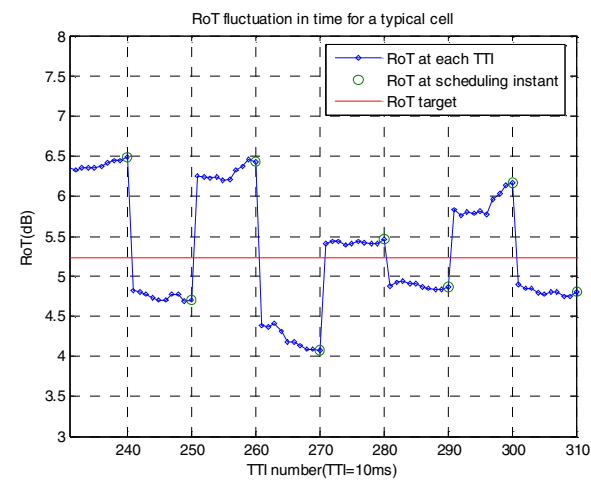

Figure 1. Typical RoT fluctuation (in time) in traditional scheduling due to intercell interference in a cell

RoT of a cell dramatically increases to well above the threshold and rapidly decreases to well below the threshold in the next scheduling instant, which highlights the fact that lack of information about neighbouring cells causes a negative impact in terms of interference outage which in turn increases the probability of packet errors. Fig. 2 shows a typical cumulative distribution of RoT at a BS.

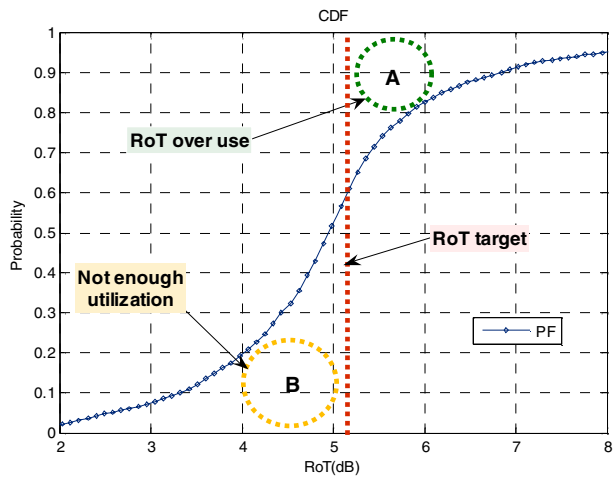

Figure 2. Resource Utilization interpretation of RoT
The ideal performance in terms of interference management would be to keep measured RoT as close to the threshold line as possible. In Fig. 2, the area marked by "A" shows instances when resources has been allocated more than allowed and " $\mathrm{B}$ " marks other instances when there are unused resources that could have been allocated to users. With a similar approach, the user average transmission rate over the simulation period provides an indication of fairness for an algorithm.

The main objective in this paper is to evaluate the performance of traditional scheduling with and without LM and also to achieve an optimised priority function for Load Matrix compared with traditional schedulers. The performance evaluation for RR, Max C/I, PF schedulers with and without LM as well as the proposed LM priority will be explained and compared in the next section.

\section{Scheduling Algorithms vs. LOAD MATRIX}

Scheduling algorithms have different objectives. Their performance depends also on the deployed system and the environment characteristics. Some algorithms, for instance, aim for fairness in resources given to the user whereas others are more focused on generating higher throughput.

\section{A. Traditional Schedulers}

Three main scheduling algorithms are considered here as the basis for analyse namely Round Robin (RR), Max C/I and Proportional Fair (PF). It is also worth noting that these schedulers are deployed here in decentralized manner same as in [2].

$\mathrm{RR}$ is a fair and simple algorithm. Resources are allocated to users in a cyclic order offering fair resource sharing among them. However the property of not considering the radio channel condition produces very low throughput. On the contrast to RR, Max C/I is based on the channel conditions by allocating the available resources to the user with the best channel quality in terms of Signal to Interference (SIR) ratio, and therefore increases the total system throughput. As a result, the users close to BS are more likely to have always better channel condition and therefore consume the resources. Max $\mathrm{C} / \mathrm{I}$ increases the cell capacity but suffers from poor fairness.

$\mathrm{PF}$ increases the influence of previous transmission rates and allows trade-off between fairness and throughput:

$$
S_{k}=\lim _{T \rightarrow \infty} \frac{1}{T} \sum_{t=1}^{T} r_{k}(t)
$$

where $r_{k}(t)$ is the transmission rate of user $k$ at time $t$ and $S_{k}$ is the average rate transmitted already. User $k$ priority can be defined as:

$$
\text { PRIORITY }_{k}=\frac{R_{k}^{\max }}{r_{k}}
$$

where $R_{k}^{\max }$ is the maximum rate allowed based on user's buffer size and power constraint.

$r_{k}$ can be evaluated through smoothed average such as [2]:

$$
r_{k}(t+1)=\left(1-\frac{1}{T c}\right) r_{k}(t)+\frac{1}{T c} R_{k}
$$


with $T c$ being the time constant of the averaging filter. With bigger $T c$, we expect better exploitation of multi-user diversity at the expense of longer packet delays. PF however tends to always select users with limited fading variation [3].

Recently proposed Score-Based (SB) scheduling algorithm [4] analyses the user's traffic performance and allocates a transmission rate according to the score measured. It provides fairness according to rate statistics and increases robustness to the asymptotic channel condition. While in PF the prioritization of transmission rate is based on own average throughput, SB takes advantage of rate statistics but not necessarily the transmission rate itself.

\section{B. Load Matrix}

On the contrary to traditional schedulers, Load Matrix concept proposed in [5] takes the intercell interference information into account in order to avoid RoT outage. LM uses a database containing the load contribution of all active users in the network. A centralized scheduler assigns radio resources to all active users in the network. We assume the averaged channel gains (over the scheduling period) from users to BSs are known to scheduler prior to rate assignment. In a network of $M$ users and $N$ cells, $L M_{i, j}$ is the load contribution by user $_{\mathrm{i}}$ in cell $_{\mathrm{j}}$ defined as:

$$
L M_{i, j}=\frac{P_{i} G_{i, j}}{N^{\prime}+\sum_{k=1}^{M} P_{k} G_{k, j}}
$$

Where $P_{i}$ is the transmit power of user,$G_{i, j}$ is the channel gain from user ${ }_{\mathrm{i}}$ to $\mathrm{BS}_{\mathrm{j}}$ and $N^{\prime}$ is the thermal noise. From $L M_{i, j}$ values stored in column $j$ of LM database, RoT of cell $l_{j}$ can be written as:

$$
R o T_{j}=\frac{1}{1-\sum_{i=1}^{M} L M_{i, j}}
$$

LM can also be used in combination with traditional schedulers. We compare traditional schedulers with and without LM enhancement in which the allocation of resources is affected by the load factor of other cells as well as the own cell. The key point of these LM-enhanced algorithms is to benefit from efficient interference control mechanism provided by LM.

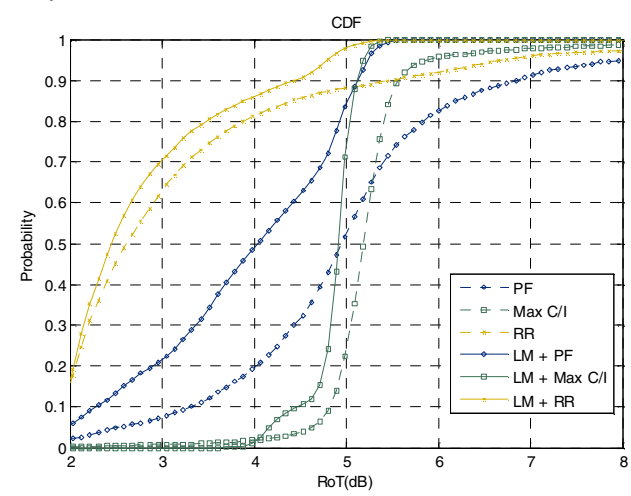

Figure 3. Cumulative distribution of RoT for traditional schedulers with and without Load Matrix enhancement
Fig. 3 compares cumulative distribution of RoT level. As expected, the main difference between the traditional and the LM-enhanced schedulers is that the probability of the RoT exceeding its target has been significantly reduced by introducing LM. LM-enhanced Max C/I has the best RoT performance over other algorithms since it has moderate use of RoT and elegantly meets the RoT restrictions. There is a high proportion of RoT well below the RoT target for LM+PF. The overall impact of the available RoT exhaustion will be explained later in this section.

When it comes to fairness observed in terms of user average throughput (see Fig. 4), they have very different characteristics. In case of Max C/I, there is a large proportion of users with very high average rate and also large proportion with significantly low rates as well compared to other schemes. RR outperforms other algorithms in terms of fairness since it provides same transmission opportunity to all users. In general, LM-enhanced schedulers show similar results in terms of fairness that have been observed for traditional schedulers with some fairness improvement in case of LM+PF.

Regarding average cell throughput shown in Fig. 5, Max $\mathrm{C} / \mathrm{I}$ has the best throughput performance which is around $30 \%$ more than PF and about $70 \%$ more than RR. By introducing Load Matrix, the average cell throughput of Max $\mathrm{C} / \mathrm{I}$ has increased by $15 \%$ while that of PF has slightly decreased due to the fact that it achieved more fairness as a trade-off. The RR shows no change in throughput over the change of interference management mechanism.

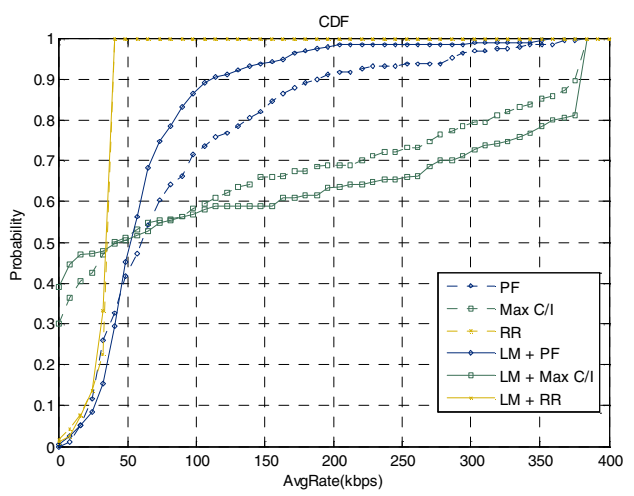

Figure 4. CDF of user average transmission rate for LM-enhanced schedulers

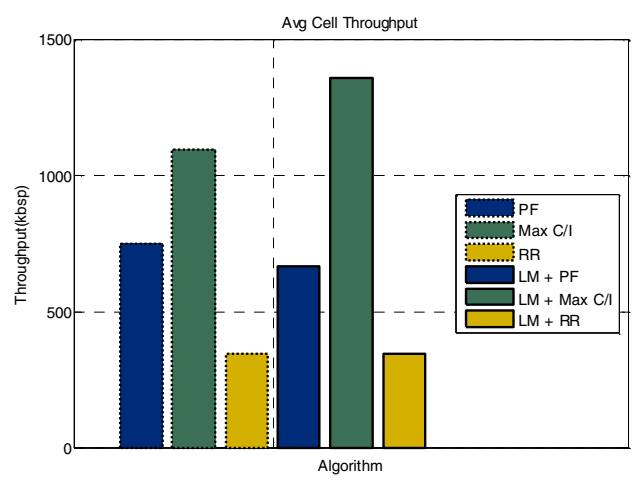

Figure 5. Average cell throughput for LM-enhanced schedulers 


\section{1) LM with Global Proportional Priority (GPP)}

The principle of the Global Proportional Priority (GPP) function [5] is highly relevant to Max C/I i.e. a user with higher channel gain has higher priority. The main difference is that GPP considers interference contribution of the user to other cells as well. If a user has good channel to more than one BS, it will not be given the rate that could have been given in traditional Max C/I. GPP function is defined as:

$$
\text { PRIORITY }_{i}=\frac{G_{i, j}}{\sum_{n=1, n \neq j}^{N} G_{i, n}}
$$

where $G_{i, j}$ is the channel gain from user ${ }_{\mathrm{i}}$ to $\mathrm{BS}_{\mathrm{j}}$ averaged over the scheduling period. It is shown in [5] that Load Matrix with GPP benefits from 30\% improvement in overall throughput over the benchmark PF algorithm (as in [2]). Also 95\% of the packets experience delay of less than 40 TTI (Transmission Time Interval) compared with 200 TTI in the benchmark PF.

\section{2) LM with Global Proportional Fair (GPF)}

Here we examine a new priority approach to Load Matrix called the Global Proportional Fair (GPF). The key property of GPF is to exploit Load Matrix in order to minimize the interference generated in a cell towards its neighbours while enhancing its fairness performance to users in the network:

$$
\begin{gathered}
\text { PRIORITY }_{i}=\frac{G_{i, j}}{\sum_{n=1, n \neq j}^{N} G_{i, n}} \cdot \frac{1}{r_{k}} \\
r_{k}=\frac{1}{W} \sum_{w=1}^{W} r_{w}
\end{gathered}
$$

where $r_{k}$ is the average user $k$ rate over time window of $W$ and $r_{w}$ is the instantaneous user rate recorded over time. In the following section, the performance of GPF in terms of throughput, fairness and also range dependency are presented in details and compared with other algorithms.

\section{Simulation Results with GPF}

In this section, the performance of GPF is evaluated and compared against LM results presented in section III. A HSUPA system simulator is used in a simulation scenario consisting of 19 cells (wrapped around) with 10 users in each cell randomly and uniformly distributed. The RoT target is set to $5.23 \mathrm{~dB}$ (i.e. $70 \%$ load factor). Other simulation parameters are presented in Table 1. Fig. 6 illustrates the basic strength of LM regardless of the algorithm being used and that is the capability to maintain RoT not to exceed its target. Similar to PF scheduler, the performance of GPF algorithm depends on the size of its averaging size $W$ as defined in (8). However, from interference outage point of view, GPF like any other LM scheduler has strict control over interference generated and RoT has been maintained below the target, regardless of the $W$ size.

It is observed in Fig. 5 that $\mathrm{LM}+\mathrm{Max} \mathrm{C} / \mathrm{I}$ provides about $15 \%$ more average cell throughput against the traditional Max
$\mathrm{C} / \mathrm{I}$ and more than $30 \%$ compared to traditional PF. Here, Fig. 8 shows that GPF produces $15 \%$ less average cell throughput compared with $\mathrm{LM}+\mathrm{Max} \mathrm{C} / \mathrm{I}$ which makes it as high in throughput as traditional Max C/I. This is whilst its fairness performance outperforms Max C/I significantly as shown in Fig. 7. That means GPF is capable of combining performance benefits of the two, i.e. the throughput advantage of Max C/I and fairness advantage of PF. No need to mention that average cell throughput in GPF is much higher than RR or PF as shown in Fig. 8.

The window size $W=10$ is selected for GPF as an example to examine the performance. The size of $W$ can swing GPF performance between throughput and fairness. Better fairness can be achieved by increasing the size of $W$ while better throughput can be produced by decreasing it.

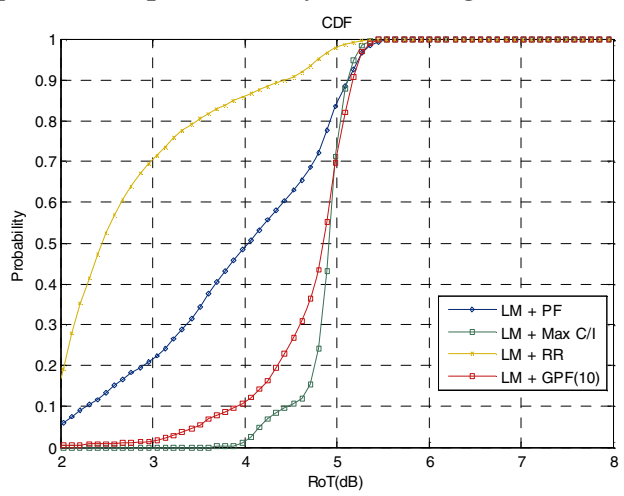

Figure 6. Cumulative distribution of RoT for LM-enhanced schedulers

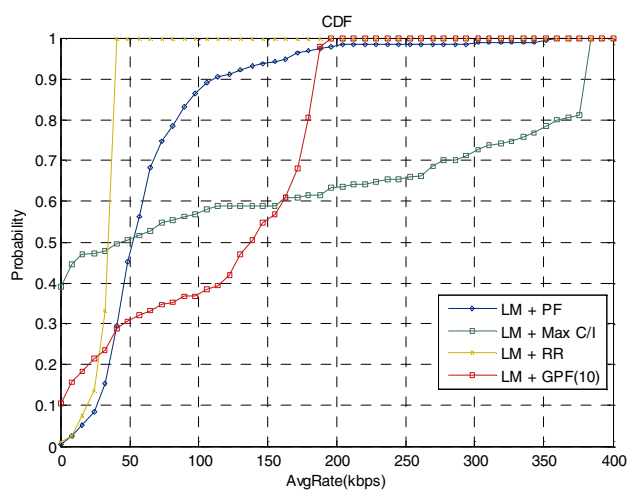

Figure 7. CDF of user average transmission rate for LM-enhanced schedulers

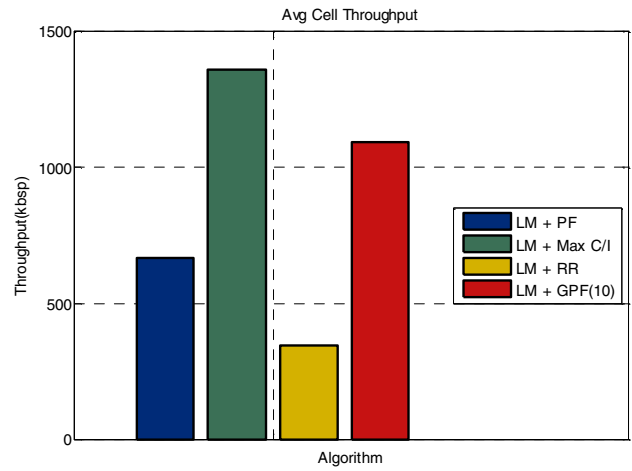

Figure 8. Average cell throughput for LM-enhanced schedulers 
Fig. 9 illustrates another important aspect of fairness and that is the throughput vs. range performance for various $W$ sizes. The higher is the window size, the better becomes the cell-edge throughput and range fairness (although it comes at a price of overall throughput). It is therefore very important to make the right balance between throughput and fairness. It is also important to provide fair chance of transmission resources with respect to the user location in the cell which is called range fairness.

Finally, Fig. 10 compares the throughput vs. range performance for Max C/I, PF and GPF $(W=20)$. One can see that GPF outperforms Max C/I in terms of fairness over the range, most importantly at cell-edge, and provides higher throughput than PF over the whole cell range.

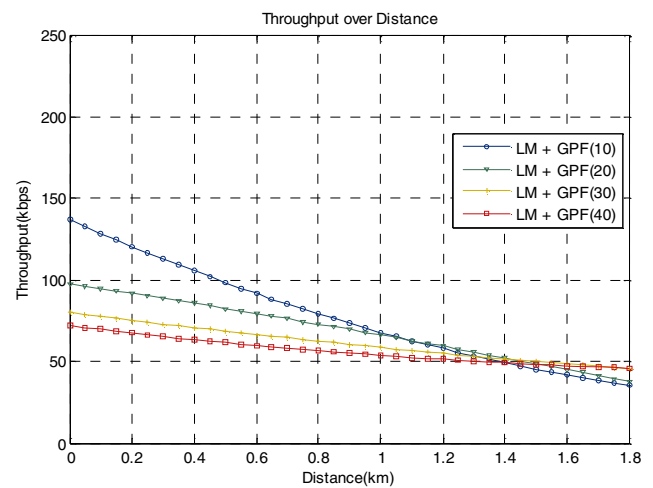

Figure 9. Throughput over distance (range fairness): effect of $W$ size

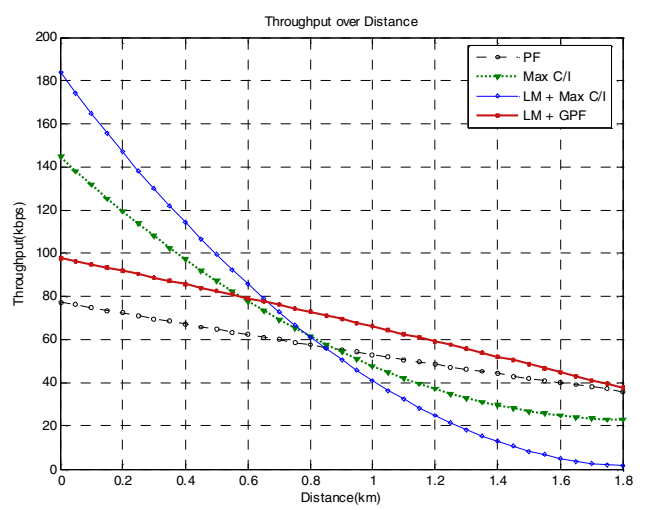

Figure 10. Throughput over distance (range fairness)

\section{CONCLUSIONS}

In this paper a novel approach towards efficient uplink scheduling is presented. Firstly the importance of resource allocation mechanisms and problems in exiting schedulers are discussed, and some of traditional schedulers are investigated.
We then assess the performance of Load Matrix scheduling combined with traditional schedulers and evaluate LMenhanced schedulers. The main reason for developing these LM-enhanced algorithms is to benefit from efficient interference control mechanism provided by LM. The effect on the scheduling performance can be observed in the simulation results provided.

\section{REFERENCES}

[1] J.M. Holtzman, "CDMA forward link waterfilling power control," in Proc. of IEEE VTC 2000-spring, vol. 3, pp. 1663-1667, spring 2000.

[2] 3GPP R1-031246, Reference Node-B Scheduler for EUL, Qualcomm Europe, www.3 gpp.org, November 2003.

[3] J.M. Holtzman, "Asymptotic analysis of proportional fair algorithm," in Proc. of 12th IEEE International Symposium on Personal, Indoor and Mobile Radio Communications 2001, vol. 2, pp. 33-37, 2001.

[4] T.Bonald, "A Score-Based Opportunistic Scheduler for Fading Radio Channels," in Proc. of European Wireless Conference, Barcelona, 2004.

[5] M.Abaii, Yajian Liu, R.Tafazolli., "An Efficient Resource Allocation Strategy for Future Wireless Cellular Systems," IEEE Trans. Wireless Communications, submitted for publication, April 2006.

[6] Harri Holma and Antti Toskala, HSDPA / HSUPA for UMTS, John Wiley \& Sons, 2006

[7] Wei Yu, Wonjong Rhee, Stephen Boyd, John M. Cioffi, "Iterative Water-Filling for Gaussian Vector Multiple-Access Channels," IEEE Trans. on Information Theory, vol.50, January 2004

[8] Andrea Goldsmith, Wireless Communications, Cambridge, 2005.

[9] 3GPP R1-031004, Example of Rel-99 TFC control algorithm, Qualcomm Europe, www.3gpp.org, October 2003.

TABLE I. SIMULATION PARAMETERS

\begin{tabular}{|l|l|}
\hline \multicolumn{1}{|c|}{ Parameter } & \multicolumn{1}{c|}{ Explanation } \\
\hline System Layout & $\begin{array}{l}\text { Hexagonal grid, omni sites, } \\
\text { 3tiers(19 base stations) wrap around }\end{array}$ \\
\hline Number of users & $190(10$ users per cell) \\
\hline Cell Radius R & $1.8 \mathrm{~km}$ \\
\hline Path loss model & $\mathrm{L}=128.1+37.6 \mathrm{Log} 10(\mathrm{R})$ \\
\hline Channel & AWGN + shadowing \\
\hline $\begin{array}{l}\text { Correlation distance of } \\
\text { slow fading }\end{array}$ & $50 \mathrm{~m}$ \\
\hline BS antenna gain & $14 \mathrm{dBi}$ \\
\hline Carrier Frequency & $2 \mathrm{Ghz}$ \\
\hline Rx antenna & 1 \\
\hline User antenna gain & $0 \mathrm{dBi}$ \\
\hline Maximum User EIRP & $21 \mathrm{dBm}$ \\
\hline Maximum BS EIRP & $24 \mathrm{dBm}$ \\
\hline CL Power control & $1 \mathrm{~dB} \mathrm{step} \mathrm{size}$ \\
\hline Transmission rate (kbps) & $8,16,32,64,128,256,384$ \\
\hline TTI & $10 \mathrm{~ms}$ \\
\hline Scheduling period & Every 10 TTI \\
\hline Traffic model & Full buffer, video streaming (3GPP H.263) \\
\hline Simulation time & $20 \mathrm{~s}$ \\
\hline RoT target & $5.23 \mathrm{~dB}(=70 \%$ load factor) \\
\hline & \\
\hline
\end{tabular}

